\title{
Resistance to ciprofloxacin in pathogenic Enterobacteriaceae in England and Wales in 1996
}

\author{
E J Threlfall, A Graham, T Cheasty, L R Ward, B Rowe
}

\begin{abstract}
In 1996, 6\% of Escherichia coli from extraintestinal infections were resistant to ciprofloxacin with minimum inhibitory concentrations (MICs) $\geqslant 2 \mathrm{mg} / \mathrm{l}$ (high level resistance). Low level resistance (MIC 0.125-1 mg/l) was also identified in $7 \%$ of Salmonella typhi, $4 \%$ of $S$ paratyphi $A$, and $4 \%$ of non-typhoidal salmonellas. However, resistance to ciprofloxacin was rarely identified in shigellas. For $E$ coli, physicians should be aware that treatment failures may occur when patients with invasive illness are treated with ciprofloxacin before the results of laboratory sensitivity tests are available. For salmonellas an increasing number of treatment failures have been recorded for patients infected with strains with low level resistance. Because of the increasing incidence of Enterobacteriaceae with low level resistance to ciprofloxacin, it is recommended that for this group of organisms a breakpoint of $0.125 \mathrm{mg} / 1$ should be included in laboratory sensitivity tests. (F Clin Pathol 1997;50:1027-1028)
\end{abstract}

Keywords: ciprofloxacin; pathogenic Enterobacteriaceae; sensitivity testing

Ciprofloxacin is the recommended antimicrobial agent for typhoid fever and has been recommended for the treatment of invasive infections with Escherichia coli and nontyphoidal salmonellas, and for acute shigellosis if clinically indicated. The emergence of resistance to this antimicrobial agent has been reported in several countries in a wide range of

Accepted for publication 10 October 1997 enterobacterial pathogens. ${ }^{1-4}$ Should it be necessary to treat invasive disease before the results of laboratory sensitivity tests are known, a knowledge of the current incidence of resistance to ciprofloxacin in such organisms may be advantageous. In this communication we report the occurrence of resistance to ciprofloxacin in extraintestinal $E$ coli, salmonella, and shigella isolated from patients in England and Wales in 1996.

\section{Materials and methods}

Strains of $E$ coli, salmonella, and shigella isolated from patients in England and Wales in 1996 and referred to the Laboratory of Enteric Pathogens for identification and typing were investigated. None of the isolates had been preselected on the basis of antimicrobial susceptibility. All isolates were tested by an agar dilution method for resistance to ampicillin, chloramphenicol, gentamicin, kanamycin, streptomycin, sulphonamides, tetracyclines, trimethoprim, furazolidone, and ciprofloxacin. ${ }^{5}$ For ciprofloxacin, the levels of antibiotic incorporated into the plates were $0.125 \mathrm{mg} / \mathrm{l}$ and $1 \mathrm{mg} / \mathrm{l}$; isolates resistant at $1 \mathrm{mg} / 1$ were subjected to full minimum inhibitory concentration (MIC) determination.

\section{Recults}

Table 1 summarises the incidence of resistance to ciprofloxacin in extraintestinal $E$ coli, salmonella, and shigella from patients in England and Wales in 1996.

Twenty three of $368(6 \%)$ isolates of $E$ coli were resistant to ciprofloxacin with MICs ranging from $2-256 \mathrm{mg} / 1$ (high level resistance). All ciprofloxacin resistant isolates were also resistant to at least one additional antimicrobial agent and 21 were multiresistant (to four or more agents). Low level resistance to ciprofloxacin (MIC $0.25-1 \mathrm{mg} / \mathrm{l}$ ) was not detected.

$S$ typhi isolates from 14 of 210 patients ( $7 \%$ ) were resistant to ciprofloxacin with MICs $0.25-1 \mathrm{mg} / \mathrm{l}$. Six of $137(4 \%) S$ paratyphi A isolates were ciprofloxacin resistant at similar levels. Nine of the 14 ciprofloxacin resistant $S$ typhi were also resistant to chloramphenicol, ampicillin, trimethoprim, streptomycin, sulphonamides, and tetracyclines but none of the
Salmonella

$S$ typhi

$S$ paratyphi A

Non-typhoidal

Shigella
210

137

30075
1043
$23(6 \%)$

$14(7 \%)$

$6(4 \%)$

$322(4 \%)$
$8(0.8 \%)$
$14(7 \%)$

$6(4 \%)$

$1315(4 \%)$ $8(0.8 \%)$
0

7 (< $0.1 \%)$ 
isolates of $S$ paratyphi A were resistant to other agents. All patients with ciprofloxacin resistant $S$ typhi and $S$ paratyphi A had a history of recent return from countries in the Indian subcontinent. For non-typhoidal salmonellas 1322 of $30075(4 \%)$ isolates had ciprofloxacin MICs ranging from $0.25-1 \mathrm{mg} / \mathrm{l}$ (low level resistance) but only seven $(<0.1 \%)$ showed high level resistance. Ciprofloxacin resistance was identified in 31 non-typhoidal serotypes but was most common in the zoonotic serotypes $S$ typhimurium, $S$ hadar, and $S$ virchow. ${ }^{6}$ Of particular concern was an increase from $7 \%$ to $14 \%$ in the incidence of ciprofloxacin resistance in multiresistant $S$ typhimurium definitive phage type (DT) 104 , which has been the second most common salmonella strain in human infections in England and Wales since 1992 and is currently the most common strain in cattle and poultry in the UK. Of the seven non-typhoidal isolates with high level resistance, none were DT 104. Three patients from whom strains with high level resistance were isolated had a history of recent foreign travel.

Only eight of $1043(0.8 \%)$ shigella isolates were resistant to ciprofloxacin (MICs $0.25-$ $1 \mathrm{mg} / \mathrm{l})$. The majority of ciprofloxacin resistant isolates were from patients who had recently returned from abroad.

\section{Discussion}

The expected serum concentration of ciprofloxacin during treatment at recommended doses is $\geqslant 2 \mathrm{mg} / 1$. In $1996,6 \%$ of $E$ coli isolates from extraintestinal infections were ciprofloxacin resistant and in all cases the level of resistance was in the range of $2-256 \mathrm{mg} / \mathrm{l}$. As $E$ coli is the most common cause of bacteraemia in England and Wales, ${ }^{7}$ physicians should be aware of the possibility of treatment failures if it is necessary to start treatment with ciprofloxacin before the results of laboratory sensitivity tests are available. It is also concerning that the majority of ciprofloxacin resistant isolates of $E$ coli from extraintestinal infections were resistant to at least four other antimicrobial drugs.

Although $7 \%$ of isolates of $S$ typhi, $4 \%$ of $S$ paratyphi A, and $4 \%$ of non-typhoidal isolates were ciprofloxacin resistant, the level of resistance in all but seven non-typhoidal isolates was in the range of $0.25-1 \mathrm{mg} / 1$. It is noteworthy that an increasing number of treatment failures have been recorded both for infections with $S$ typh $i,{ }^{8}$ and for invasive infections with non-typhoidal salmonellas'; in all cases the ciprofloxacin MICs of the strains have been in the range $0.25-1 \mathrm{mg} / 1$.

A substantial increase in the incidence of ciprofloxacin resistance in non-typhoidal salmonellas has followed the licensing in 1993 for use in animal husbandry in the UK of the related fluoroquinolone antibiotic enrofloxacin. This agent has subsequently been extensively used for treatment and prophylaxis in both cattle and poultry.

Resistance to ciprofloxacin is mediated either by chromosomal mutations in the gyrA gene, or by decreased cell wall permeability. Such mutations confer resistance not only to ciprofloxacin but also to the entire class of fluoroquinolone agents. As the majority of infections caused by non-typhoidal salmonellas in England and Wales are zoonotic in origin, it is possible that the use of fluoroquinolone antibiotics in animal husbandry has contributed to the upsurge of ciprofloxacin resistance in nontyphoidal salmonellas. In this respect it is regrettable that the effectiveness of ciprofloxacin in human medicine is being eroded by the use of related fluoroquinolone antibiotics in animal husbandry. Resistance to ciprofloxacin remains uncommon for shigella $(<1 \%$ of isolates) and should antimicrobial treatment for acute shigellosis be indicated, ciprofloxacin remains a useful option.

The breakpoints used by many laboratories in testing for ciprofloxacin sensitivity in Enterobacteriaceae are $1 \mathrm{mg} / \mathrm{l}$ (low level) and $4 \mathrm{mg} / 1$ (high level). ${ }^{10}$ Using these levels the reduced susceptibility of the isolates with MICs in the range $0.25-1.0 \mathrm{mg} / 1$ would not be appreciated and treatment failures could follow. When testing for resistance to ciprofloxacin by breakpoint for enterobacteria, we recommend using $0.125 \mathrm{mg} / \mathrm{l}$ supported by full MICs for isolates resistant at this level. For disc testing, we endorse the recent recommendations of Murphy et al who considered that because borderline susceptibility in vitro may predict treatment failure, the full MICs of isolates resistant at $\geqslant 0.25 \mathrm{mg} / 1$ on disc testing should be determined. ${ }^{2}$

1 Threlfall EJ, Cheasty T, Graham A, Rowe B. High-level resistance to ciprofloxacin in Escherichia coli. Lancet 1997;349:403.

2 Murphy OM, Marshall C, Stewart D, Freeman R. Ciprofloxacin-resistant Enterobacteriaceae. Lancet 1997; 349:1028-9.

3 Frost JA, Kelleher A, Rowe B. Increasing ciprofloxacin resistance in salmonellas in England and Wales, 19911994. F Antimicrob Chemother 1996;37:85-91.

4 Everett MJ, Jin YF, Ricci V, Piddock LVJ Contributions of individual mechanisms to fluoroquinolone resistance in 36 Escherichia coli strains isolated from humans and animals. Antimicrob Agents Chemother 1996;40:2380-6.

5 Frost JA. Testing for resistance to antibacterial drugs. In Chart $\mathrm{H}$, ed. Methods in practical laboratory bacteriology. New York: CRC Press, 1994:73-82.

6 Threlfall EJ, Ward LR, Skinner JA, Rowe B. Increase in antibiotic resistance in nontyphoidal salmonellas from humans in England and Wales: a comparison of data for 1994 and 1996. Microb Drug Resist 1997;3:263-6.

7 Anon. Bacteraemia: 1994,1995 and 1996. Commun Dis Rep CDR Wkly 1997;7:14.

8 Umasankar S, Wall RA, Berger J. A case of ciprofloxacinresistant typhoid fever. Commun Dis Rep CDR Rev 1992;2: R139-40.

9 Piddock LJV, Whale K, Wise R. Quinolone resistance in salmonella; clinical experience. Lancet 1990;335:1459.

10 Anon. Supplementary report by the working party on antimicrobial sensitivity testing by the British Society for Antimicrobial Chemotherapy. $f$ Antimicrob Chemother 1996;38: 1103-5. 